\title{
Anastomotic branch from the median nerve to the musculocutaneous nerve: a case report
}

\author{
Feray Güleç Uyaroğlu ${ }^{1}$, Gülgün Kayalığlu², Mete Ertürk ${ }^{2}$ \\ ${ }^{1}$ Department of Neurology, Ege University Faculty of Medicine, Izmir, Turkey \\ ${ }^{2}$ Department of Anatomy, Ege University Faculty of Medicine, Izmir, Turkey
}

\begin{abstract}
Anomalies of the brachial plexus and its terminal branches are not uncommon. Communicating branch arising from the musculocutaneous nerve to the median nerve is a frequent variation, whereas the presence of an anastomotic branch arising from the median nerve and joining the musculocutaneous nerve is very rare. During routine dissection of cadaver upper limbs, we observed an anastomotic branch arising from the median nerve running distally to join with the branches of the musculocutaneous nerve in a left upper extremity. The anastomotic branch originated from the median nerve $11.23 \mathrm{~cm}$ proximal to the interepicondylar line. After running distally and coursing between the biceps brachii and the brachialis muscles, this anastomotic branch communicated with two branches of the musculocutaneous nerve separately. The presence of the communicating branches between these nerves should be considered during surgical interventions and clinical investigations of the arm.
\end{abstract}

Key words: anatomy; brachial plexus; communicating branch; upper limb; variation

Anatomy 2008; 2: 63-66, @ 2008 TSACA

\section{Introduction}

Variations of the brachial plexus and its terminal branches are common. Nerves of the brachial plexus are formed and remain in anterior and posterior planes of the arm. ${ }^{1}$ Musculocutaneous and median nerves are in the anterior plane, like the ulnar nerve, medial cutaneous nerves of the arm and forearm, and the medial and lateral pectoral nerves. The median nerve, formed by the union of the terminal branch of the lateral $(\mathrm{C} 5, \mathrm{C} 6, \mathrm{C} 7)$ and medial $(\mathrm{C} 8, \mathrm{~T} 1)$ cords of the brachial plexus, enters the arm lateral to the brachial artery and to the forearm between the two heads of the pronator teres muscle. The median nerve does not give motor branches in the arm, but gives off vascular branches to the brachial artery and supplies articular branches to the elbow joint. If the lateral root of the median nerve is small, the musculocuta- neous nerve (C5, C6, and C7) connects with the median nerve in the arm. ${ }^{2,3}$ The musculocutaneous nerve is the continuation of the lateral cord of the brachial plexus. It pierces the coracobrachialis muscle and descends laterally between the biceps and brachialis muscles and supplies all the muscles in the anterior (flexor) compartment of the arm.

The musculocutaneous nerve has frequent variations associated with its connection to the median nerve: it may run behind the coracobrachialis muscle or adhere for some distance to the median nerve and pass behind the biceps brachii muscle; some fibers of the median nerve may run in the musculocutaneous nerve; less frequently the median nerve sends a branch to the musculocutaneous nerve. ${ }^{2,3}$ The presence of a communicating branch arising from the musculocutaneous nerve and joining to the median nerve is more common than the 
presence of a communicating branch arising from the median nerve joining to the musculocutaneous nerve. The present paper deals with a rare variation of the musculocutaneous and median nerves, an anastomotic branch originating from the median nerve and joining to the musculocutaneous nerve.

\section{Case Report}

During the routine anatomy dissections, we observed an anastomotic branch arising from the median nerve and running distally to join with the branches of the musculocutaneous nerve in the left upper extremity of a male cadaver from the collection in Department of Anatomy, Ege University Faculty of Medicine (Figure 1). The musculocutaneous nerve divided into two branches at a point $14.44 \mathrm{~cm}$ proximal to the interepicondylar line. The anastomotic branch originating from the median nerve at $11.23 \mathrm{~cm}$ proximal to the interepicondylar line ran distally and coursed between the biceps brachii and the brachialis muscles, than communicated with the two branches of the musculocutaneous nerve separately. The length of the anastomotic branch was $4.53 \mathrm{~cm}$ from the arising point to its bifurcation point, and $9.7 \mathrm{~mm}$ and $9.2 \mathrm{~mm}$ from its bifurcation point to the communication point to the two branches (Figure 2).

\section{Discussion}

Anomalies of the brachial plexus and its terminal branches are common. Communications between the median and musculocutaneous nerves have been reported in the literature. Communicating branches arising from the musculocutaneous nerve and joining to the median nerve are observed frequently. On the contrary, anastomotic branches arising from the median nerve and than joining the musculocutaneous nerve as in this case are rare.

Various authors examined connections between the median and musculocutaneous nerves, the incidence of such a connection ranges from $5 \%$ to $63.5 \%$. In the study of Choi et al., connections of the median and musculocutaneous nerves were observed in $26.4 \%$ of 138 arms. The nerves were either fused, or there were one or two communicating branches from the musculocutaneous nerve to the median nerve. ${ }^{4}$ Venieratos and Anagnostopoulou reported 22 connections (13.9\%) between the median and musculocutaneous nerves in 158 upper extremities they have studied. The origin of the connection was frequently distal to the coracobrachialis muscle as in our case. ${ }^{5}$ In the study of Prasada Rao and Chaudhary, on 24 upper arms, communication from the musculocutaneous nerve to the median nerve was observed in $33 \%$, however no anastomotic branch

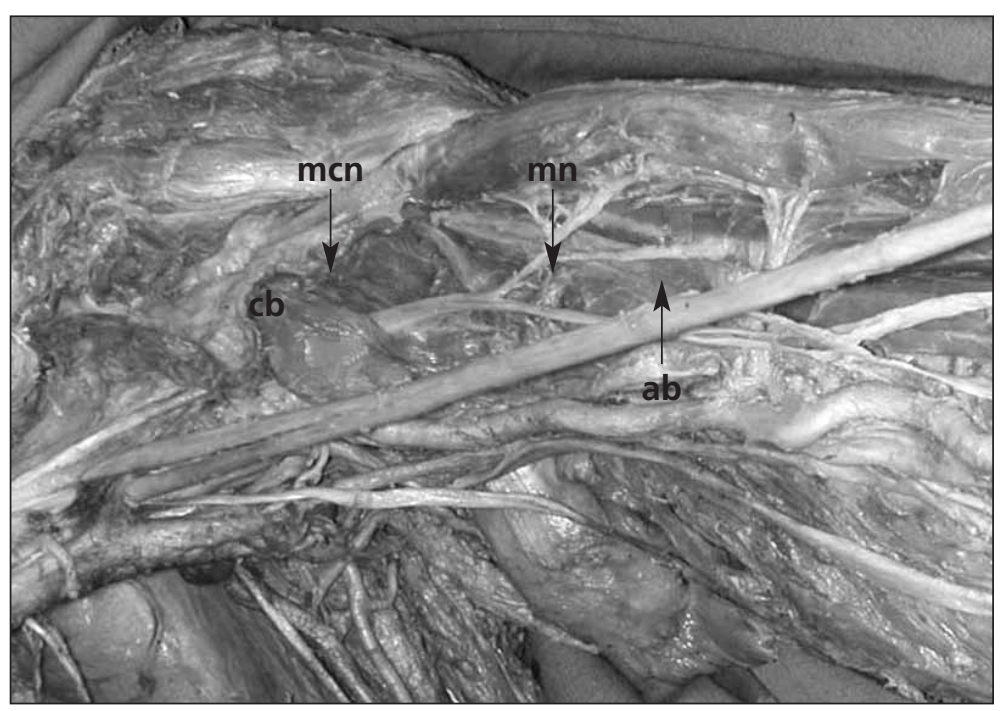

Figure 1. Anastomotic branch originating from the median nerve in the left upper limb. mcn: musculocutaneous nerve; mn: median nerve; cb: coracobrachialis muscle; ab: anastomotic branch. 
Figure 2. Connection between the median nerve to the musculocutaneous nerve in the arm. mn: median nerve; mcn: musculocutaneous nerve; ab: anastomotic branch; ba: brachial artery; bb: biceps brachii muscle.

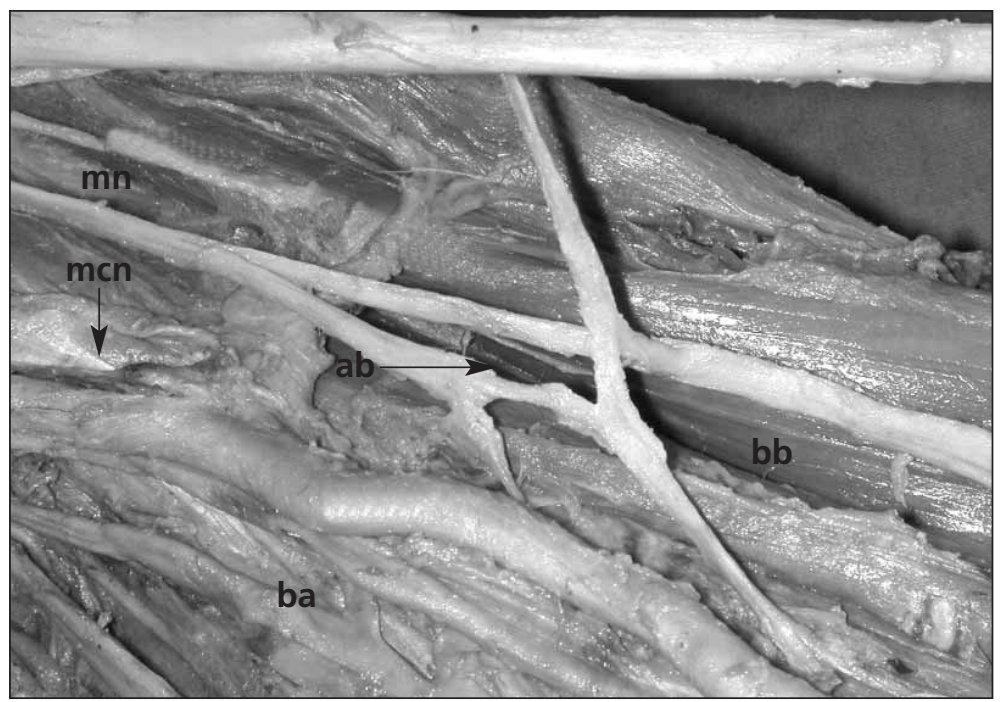

from the median nerve to the musculocutaneous nerve was observed. ${ }^{6}$ Loukas and Aqueelah found the communicating branch between the median and musculocutaneous nerves in $63.5 \%$ of cadavers, most of these connections located proximal to the point of entry of the musculocutaneous nerve into the coracobrachialis muscle. $^{7}$ Abu-Hijleh observed a case with three-headed biceps brachii muscle associated with duplicated musculocutaneous nerve in cadaver, in the lower arm, a distal musculocutaneous nerve arose from the median nerve. ${ }^{8}$ Sarıkçıŏlu et al. reported the presence of a superficial brachial artery, communication between the musculocutaneous and median nerves and innervation of the coracobrachialis muscle by a branch arising from the lateral cord. ${ }^{9}$

Anastomotic branches arising from the median nerve and than joining the musculocutaneous nerve as in this case are also reported. Beheiry observed the communicating branch from the median nerve to the musculocutaneous nerve in $5 \%$ of the limbs. ${ }^{10}$ Kosugi et al. reported that the presence of a supernumerary head effected the course and branching of the musculocutaneous nerve. In their study, a communicating branch arising from the median nerve was found in $16 \%$ of the cases with a supernumerary head of biceps brachii muscle. ${ }^{11}$ Saeed and Rufai reported a case with the median nerve formed by the fusion of three roots, two from the lateral and one from the medial cord of the brachial plexus. In their case, the median nerve contributed a communicating branch to the musculocutaneous nerve in the left upper limb. This communicating branch arose from the median nerve $13 \mathrm{~cm}$ distal to its formation and than coursed laterally and downward for about $3 \mathrm{~cm}$ before joining the musculocutaneous nerve. ${ }^{12}$ In our case, the length of the anastomotic branch was $4.53 \mathrm{~cm}$ from the arising point to its bifurcation point, and $0.97 \mathrm{~cm}$ and $0.92 \mathrm{~cm}$ from its bifurcation point to the communication point.

Understanding the embryologic development of the brachial plexus is important in explaining the origin of possible anatomic variations. The human upper limb bud appears at 26-27 days in the developing embryo and motor axons arising from the spinal cord enter the limb buds during the fifth week. ${ }^{13}$ Formation of the brachial plexus is evident from about 34-35 days as a single radicular cone in the upper limb. At between 38 and 40 days, the major branches of the brachial plexus become visible, and the median, radial and ulnar nerves enter the hand plate. ${ }^{14,15}$ The brachial plexus is divided into ventral and dorsal segments and the ventral segment that give roots to the median and ulnar nerves. The musculocutaneous nerve arises from the median nerve. ${ }^{16-18}$ About 46-48 days, all the upper limb nerves comprise an orientation and arrangement similar to those seen in the adult. 
Anastomosis between the median and musculocutaneous nerves is attributable to the common origin of the musculocutaneous and median nerves during development.

The communicating branch arising from the median nerve to the musculocutaneous nerve was encountered very rare as the presented case of this paper. These connections and distribution patterns of both nerves are important in surgical approaches to the arm and implications of clinical findings. Surgeons and clinicians should know the presence of a communicating branch and its variations during surgical procedures and clinical investigations of the arm.

\section{References}

1. Alnot JY, Huten B. Systematisation of the brachial plexus. Rev Chir Orthop Reparatrice Appar Mot 1977; 68: 27-34.

2. Standring S. Gray's anatomy. The anatomical basis of clinical practice. 39th ed. London: Elsevier Churchill Livingstone; 2005.

3. Moore KL, Dalley AF. Clinically oriented anatomy. 4th ed. Philadelphia: Lippincott Williams \& Wilkins; 1999.

4. Choi D, Rodriguez-Niedenfuhr M, Vazquez T, Parkin I, Sanudo JR. Patterns of connections between the musculocutaneous and median nerves in the axilla and arm. Clin Anat 2002; 15: 11-7.

5. Venieratos D, Anagnostopoulou S. Classification of communications between the musculocutaneous and median nerves. Clin Anat 1998; 11: 327-31.

6. Prasada Rao PV, Chaudhary SC. Communication of the musculocutaneous nerve with the median nerve. East Afr Med 7 2000; 77: 498-503.

7. Loukas M, Aqueelah H. Musculocutaneous and median nerve connections within, proximal and distal to the coracobrachialis muscle. Folia Morphol 2005; 64: 101-8.
8. Abu-Hijleh MF. Three-headed biceps brachii muscle associated with duplicated musculocutaneous nerve. Clin Anat 2005; 18: 3769.

9. Sarıkçıŏlu L, Coşkun N, Özkan O. A case with multiple anomalies in the upper limb. Surg Radiol Anat 2001; 23: 65-8.

10. Beheiry EE. Anatomical variations of the median nerve distribution and communication in the arm. Folia Morphol 2004; 63: 313 8.

11. Kosugi K, Shibata S, Yamashita H. Supernumerary head of biceps brachii and branching pattern of the musculocutaneous nerve in Japanese. Surg Radiol Anat 1992; 14: 175-85.

12. Saeed M, Rufai AA. Median and musculocutaneous nerves: variant formation and distribution. Clin Anat 2003; 16: 453-7.

13. Moore KL, Persaud TVN. The developing human: clinically oriented embryology. 6th ed. Philadelphia: W.B. Saunders Company; 1998.

14. O'Rahilly R, Gardner E. The timing and sequence of events in the development of the limbs in the human embryo. Anat Embryol 1975; 148: 1-23.

15. Shinohara H, Naora H, Hashimoto R, Hatta T, Tanaka O. Development of the innervation pattern in the upper limb of staged human embryos. Acta Anat 1990; 138: $265-9$.

16. Windle WF, Fitzgerald JE. Development of the spinal reflex mechanism in human embryos. 7 Comp Neurol 1937; 67: 493-509 [cited In: Paxinos G, Mai JK, editors. The human nervous system. San Diego: Elsevier; 2004. p. 103.]

17. Fitzgerald JE, Windle WF. Some observations on early human fetal movements. 7 Comp Neurol 1942; 76: 159-167. [In: Paxinos G, Mai JK, editors. The human nervous system. San Diego: Elsevier; 2004. p. 103.\}

18. Iwata H. Studies on the development of the brachial plexus in Japanese embryo. Rep Dept Anat Mie Prefect Univ Sch Med 1960; 13 : 129-44.

\footnotetext{
Correspondence to: Gülgün Kayalığlu, MD

Department of Anatomy, Ege University,

Faculty of Medicine, Bornova, Izmir, TR-35100, Turkey

Phone: +90 53268609 21; Fax: +90 2323422142

e-mail: gulgun.kayalioglu@ege.edu.tr

Conflict of interest statement: No conflicts declared.
} 